\title{
Effect of different enamel conditionings on the bond strength of glass ionomer cement and ceramic brackets
}

\author{
Dauro Douglas Oliveira ${ }^{1}$, Thomas Gerard Bradley², Matheus Melo Pithon ${ }^{3}$, \\ Mariele Cristina Garcia Pantuzo ${ }^{1}$, Emílio Akaki ${ }^{1}$, Virendra Dhuru ${ }^{4}$ \\ ${ }^{1}$ Pontifícia Universidade Católica de Minas Gerais - PUC Minas, Department of Dentistry, Area of Orthodontics, Belo Horizonte, MG, Brazil \\ ${ }^{2}$ Marquette University, School of Dentistry, Department of Developmental Sciences, Area of Orthodontics, Milwaukee, WI, USA \\ ${ }^{3}$ Universidade Estadual do Sudoeste da Bahia - UESB, School of Dentistry, Department of Healthy, Area of Orthodontics, Jequié, BA, Brazil \\ ${ }^{4}$ Marquette University, School of Dentistry, Department of General Dental Sciences, Area of Dental Materials, Milwaukee, WI, USA
}

\begin{abstract}
Aim: To evaluate the effect of different enamel conditionings on the shear bond strength (SBS) and bond failure patterns of a resin modified glass ionomer cement (RMGIC) used to bond ceramic brackets. Methods: 105 human premolars extracted for orthodontic reasons were divided into 7 groups according to the enamel surface treatments: conditioners (35\% phosphoric acid and 10\% polyacrylic acid), type of adhesive (Transbond $₫$ and Fuji Ortho LC Capsule $®$ ), washing time and drying of the surface. Results: A significant $p<0.05$ was observed among the shear bond strength values obtained with seven different types of enamel preparation prior to bonding. The group that had the tooth surface conditioned with polyacrylic acid and enamel surface slightly dried before bracket placement showed no significant difference $(p>0.05)$ to the group that had brackets bonded with composite resin. The two groups that had enamel prepared with polyacrylic acid and brackets bonded with GIC on a wet surface showed significantly lower shear bond strength than the control group $(\mathrm{p}<0.05)$. Conclusions: Moisture contamination decreased SBS, but not enough to preclude the use of RMGIC as an alternative to composite resin (CR) for direct bonding of ceramic brackets.
\end{abstract}

Keywords: glass ionomer cements; shear strength; dental bonding; orthodontic brackets.

\section{Introduction}

The introduction of direct bonding was a major development in Orthodontics and bonding brackets with composite resin (CR) has been considered the standard of care for many years ${ }^{1-2}$. However, bonding on an acid-etched tooth surface presents

Received for publication: September 21, 2014 Accepted: November 25, 2014

Correspondence to: Dauro Douglas Oliveira Pontifícia Universidade Católica de Minas Gerais - PUC Minas Avenida. Dom José Gaspar, 500

Prédio 46, sala 101.

Belo Horizonte, MG,Brasil E-mail: dauro.bhe@gmail.com some disadvantages, such as enamel loss during conditioning and adhesive removal $^{3}$. Furthermore, contamination with oral fluids increases bond failure and the risk of developing caries during orthodontic therapy may be higher because the fixed appliances make an adequate oral hygiene more difficult ${ }^{4}$. Thus, biofilm levels surrounding the brackets are increased and demineralization often occurs in these areas $^{5}$. An adhesive system that provides sufficient retention to orthodontic brackets and tubes, decreasing the failure rate due to moisture contamination and preventing decalcification would be ideal.

The fluoride releasing properties of glass ionomer cement (GIC) made it an interesting material for orthodontic use. Since GIC presented similar retention 
properties as zinc phosphate and zinc polycarboxylate cements $^{6}$ and also release/uptake fluoride ${ }^{7}$, it became the material of choice for cementation of orthodontic bands ${ }^{8}$. GIC was also tested as a direct bonding agent in Orthodontics. In the early studies, conventional GIC has not shown the same effectiveness as CR to bond orthodontic brackets, but resin modified glass ionomer cements (RMGIC) have shown good potential to bond metallic brackets 9 .

There are few studies reporting the use of RMGIC for bonding ceramic brackets, especially when it is used after different enamel conditionings. This investigation evaluated the effect of different enamel conditioning procedures on the shear bond strength (SBS) and bond failure pattern (BFP) of a RMGIC used for bonding ceramic brackets.

\section{Material and methods}

The bonding agents used in this study were a CR (Transbond XT, 3M/Unitek, Monrovia, CA, USA) and a RMGIC (Fuji Ortho LC Capsule, GC America, Alsip, IL, USA). Two types of enamel conditioners were evaluated in this study, 35\% phosphoric acid (PHA) (Transbond XT Etching Gel, 3M/Unitek,) and 10\% polyacrylic acid (PAA) (Fuji Ortho Conditioner, GC America).

The ceramic premolar brackets used in this experiment were Standard Edgewise $0.022 \times 0.028$-in slot with mechanical retention on their bases (Clarity, $3 \mathrm{M} /$ Unitek).

\section{Preparation of Specimens}

One-hundred and five human premolars, extracted for orthodontic purposes, with intact labial surface and caries or restorations-free were collected and randomly assigned to 7 groups containing 15 teeth per group. The roots were sectioned and removed about $2 \mathrm{~mm}$ apically from the cementoenamel junction. A mounting fixture was fabricated to guarantee parallelism of the teeth labial surfaces and the base of the specimen holders. Two $0.022 \times 0.028$-in slot, Standard Edgewise stainless steel brackets (3M/Unitek,) were bonded to the rim of the acrylic cup (Figure 1A).

A copper end cap that had half of its volume filled with self-curing acrylic was placed on the acrylic holder. A piece of $0.021 \times 0.025$-in stainless steel wire was bent to create a $2.5 \mathrm{~mm}$ step up in the wire. A ceramic bracket identical to those used for the experiment was tied to the wire by an elastic ligature, so that it was positioned at the center of the crown, both occluso-gingivally and mesio-distally (Figure 1B) and fixed to the tooth with sticky wax (Figure 1C). Labial surfaces of the mounted premolars were cleaned and dried (Figure 1D). The following groups of enamel surface treatments were performed:

(1) CT (Control): Enamel was etched for $30 \mathrm{~s}$ with $35 \%$ PHA, rinsed for $10 \mathrm{~s}$ with tap water and dried with oilfree compressed air for $10 \mathrm{~s}$. A thin coat of primer was placed and light-cured for $10 \mathrm{~s}$.

(2) PA-W: $10 \%$ PAA for $20 \mathrm{~s}$ and rinsed with tap water

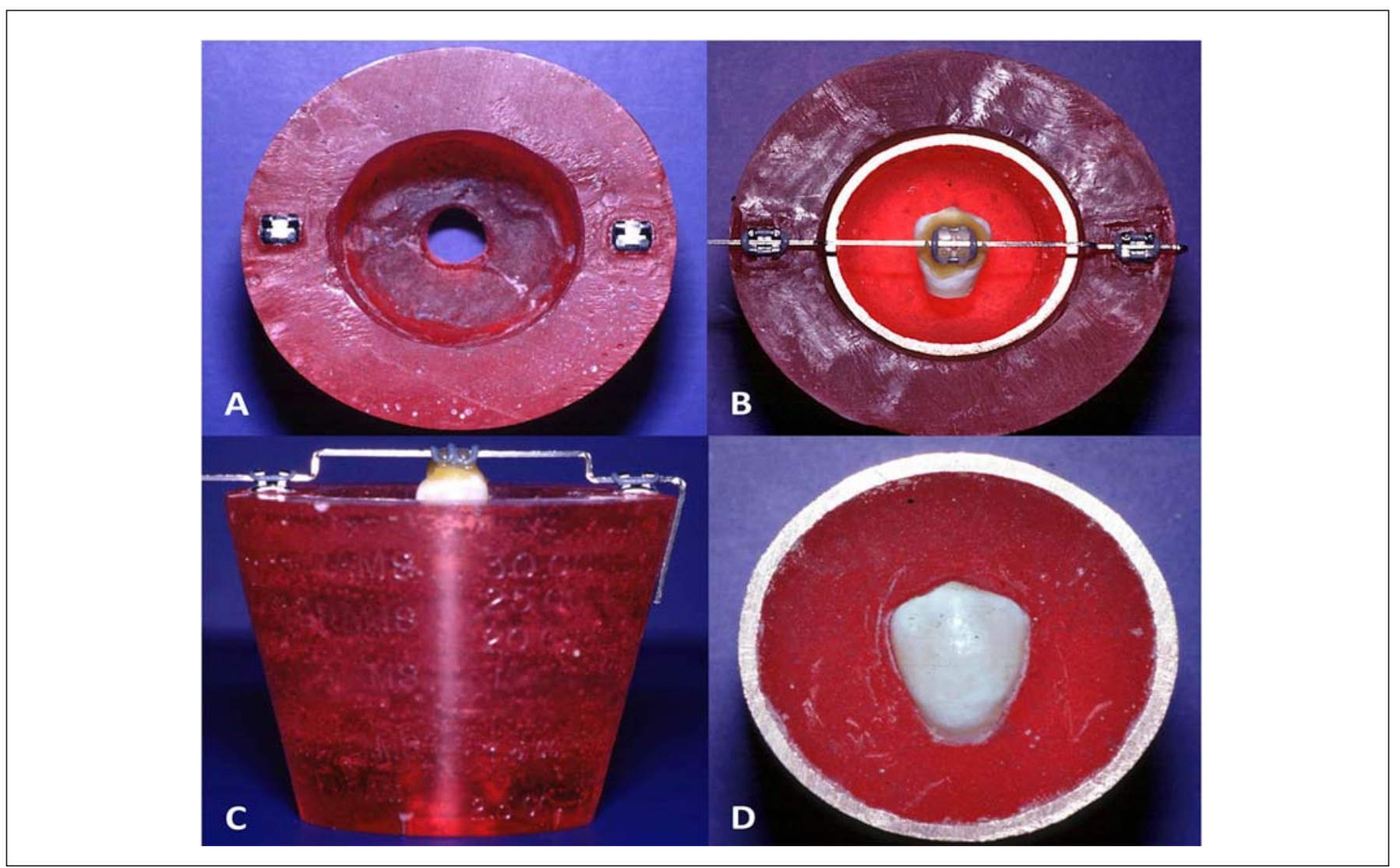

Fig. 1. Mounting fixture used to standardize sample preparation. A. Brackets bonded to the acrylic cup rim; B. Premolar suspended in the center of the copper end-cap; C. Premolar with occluso-labial surface above end-cap rim; D. Mounted premolar with labial surface exposed for bracket placement. 
for $10 \mathrm{~s}$. The bonding surfaces of the teeth were wiped with a water-moistened cotton roll.

(3) PA-D-W: $10 \%$ PAA for $20 \mathrm{~s}$, rinsed for $10 \mathrm{~s}$ with tap water and dried with oil-free compressed air for $10 \mathrm{~s}$. The surfaces were wiped with a water-moistened cotton roll.

(4) PA-LD: 10\% PAA for $20 \mathrm{~s}$ and rinsed with tap water for $10 \mathrm{~s}$. The surfaces were lightly dried with oil-free compressed air for $1 \mathrm{~s}$.

(5) PH-W: 35\% PHA for $15 \mathrm{~s}$ and rinsed with tap water for $10 \mathrm{~s}$. The surfaces were wiped with a water-moistened cotton roll.

(6) PH-D-W: 35\% PHA for $15 \mathrm{~s}$, rinsed for $10 \mathrm{~s}$ with tap water and dried with oil-free compressed air for $10 \mathrm{~s}$. The surfaces were wiped with a water-moistened cotton roll.

(7) PH-LD: 35\% PHA for $15 \mathrm{~s}$ and rinsed for $10 \mathrm{~s}$ with tap water. The surfaces were lightly dried with oil-free compressed air for $1 \mathrm{~s}$.

The brackets in group CT were bonded with CR while RMGIC was used in all other groups. Enamel conditioning in the PA-W group followed strictly the manufacturers' recommendations. The tested RMGIC was pre-proportioned in capsules and mixed by a mixing machine (GC America) to avoid possible inaccuracy of the powder/liquid ratio and to assure thorough mixing of the components. The bracket was placed at the center of the labial surface of the prepared tooth with sufficient pressure to obtain the thinnest layer of cement between the bracket and the tooth. After the removal of any excess of cement surrounding the bracket base, the cement was cured with a light unit (Demetron/Kerr, Danbury, CT, USA) for $10 \mathrm{~s}$ on each margin of the bracket at a maximum distance of $1 \mathrm{~mm}$. The light intensity was tested using the built-in radiometer to assure that the correct wavelength (above 450 $\mathrm{nm})$ was used. All samples were stored in distilled water at room temperature for $24 \mathrm{~h}$ until the test was carried out.

\section{SBS Test}

A universal testing machine (Instron Corp., Canton, MA, USA) was equipped with a $50 \mathrm{~kg}$ load cell and used for all SBS tests. The test specimens were secured in a vise clamped onto the load cell platter and the load was applied to the bracket base (Figure 2) at a crosshead speed of $0.5 \mathrm{~mm} / \mathrm{min}$.

\section{Bond Failure Analysis}

Bracket bases and corresponding enamel surfaces were evaluated with an optical stereomicroscope at $10 \mathrm{X}$ magnification. The percentage of adhesive that remained on both surfaces was quantified according to the Adhesive Remnant Index (ARI) ${ }^{10}$ : (0) no adhesive on the enamel; (1) less than $50 \%$ of the adhesive remained on the tooth; (2) more than $50 \%$ of the adhesive was left on the enamel; (3) $100 \%$ of the adhesive remained on the tooth.

\section{Results}

One-way ANOVA revealed a significant difference $(p<0.05)$ among the SBS obtained with the different enamel

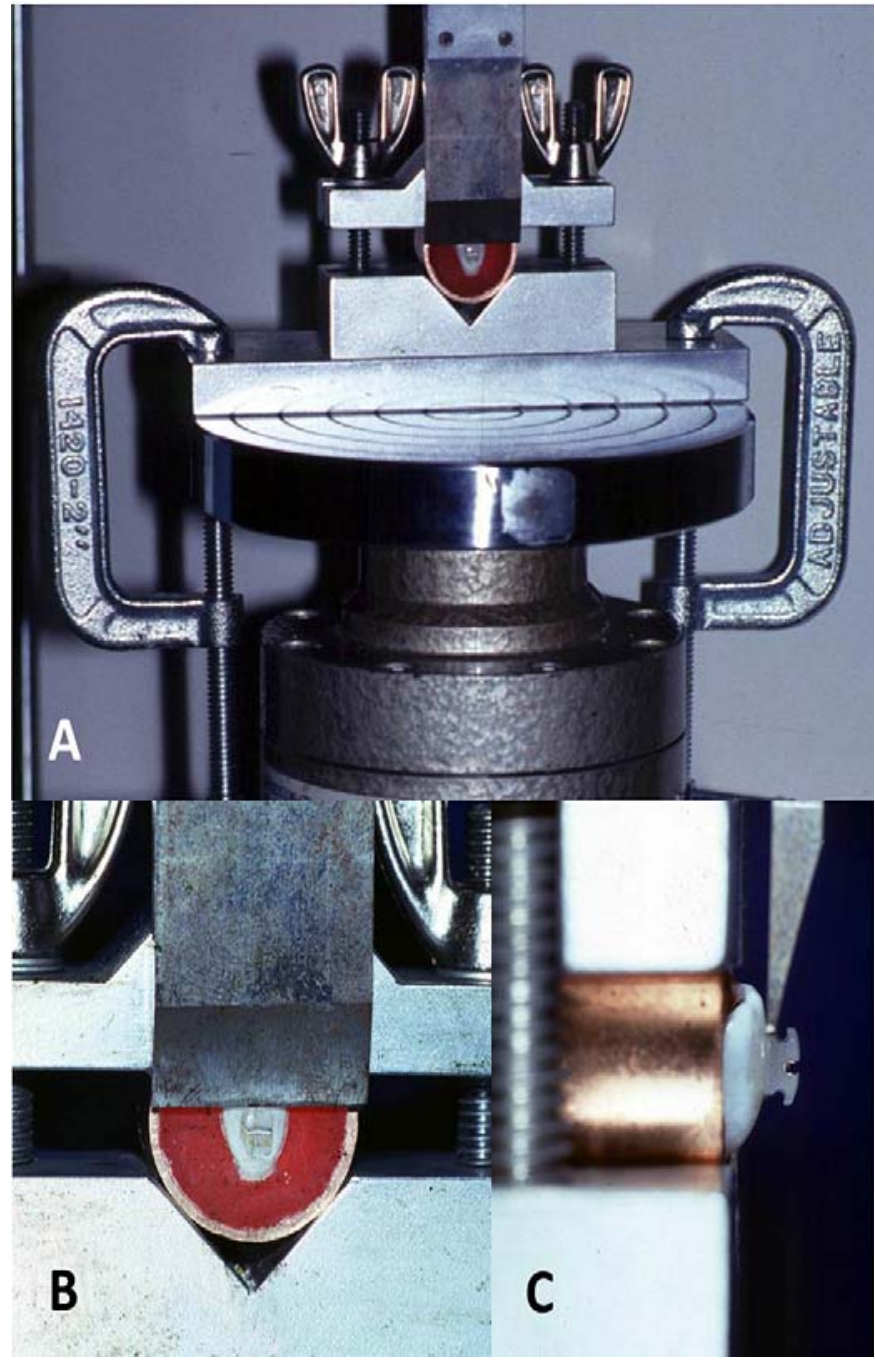

Fig. 2. SBS apparatus. A: Specimen secured in a vise clamp onto the load cell; B: Frontal close up of fixture for shear loading. C. Shear loading blade perpendicular to tooth-bracket interface.

conditionings. The mean SBS values and standard deviations (SD) for the different treatment groups were compared with Tukey Multiple Comparison Test (Table 1). It showed no statistically significant differences $(p>0.05)$ between $C T$ and all groups bonded with RMGIC that had enamel etched with PHA and the PA-LD group. However, the two groups that had enamel prepared with PAA and brackets bonded with RMGIC on a wet surface showed significantly lower SBS than CT $(p<0.05)$.

The group that followed the manufacturers' recommendations (PA-W) showed a significantly lower SBS $(p<0.05)$ than 4 out of the other 5 experimental groups $(P A-$ LD, PH-W, PH-D-W, and PH-LD). The only exception was the group that used a different moisturizing technique and that also had the tooth surface conditioned with PAA (PA-D$\mathrm{W})$. Even though the results of PA-W and PA-D-W were not significantly different $(p>0.05)$, PA-D-W showed higher SBS mean values. In consequence, PA-D-W was not significantly different $(p>0.05)$ from PA-LD and PH-D-W.

The results also indicated that there was a statistically significant difference $(\mathrm{p}<0.05)$ in the BFP among the groups. 
Table 1. Mean shear bond strength of ceramic brackets bonded with two different adhesives to enamel surface subjected to different treatments.

\begin{tabular}{lccccc}
\hline Treatment* & $\begin{array}{c}\text { Mean } \\
\text { Deviation } \\
* *(\mathrm{MPa})\end{array}$ & $\begin{array}{c}\text { Standard } \\
\text { Coefficient } \\
(\mathrm{MPa})\end{array}$ & $\begin{array}{c}\text { Variation } \\
(\%)\end{array}$ & $\begin{array}{c}\text { Minimum } \\
(\mathrm{MPa})\end{array}$ & Maximum \\
CP & $17.9^{\mathrm{c}}$ & 3.4 & 19 & 12.2 & 23.4 \\
PA-W & $10.8^{\mathrm{a}}$ & 3.9 & 36 & 4.6 & 15.6 \\
PA-D-W & $12.6^{\mathrm{ab}}$ & 3.0 & 24 & 7.5 & 16.7 \\
PA-LD & $16.2^{\mathrm{bc}}$ & 3.0 & 20 & 12.4 & 24.2 \\
PH -W & $16.4^{\mathrm{c}}$ & 3.4 & 24 & 9.0 & 24.3 \\
PH-D-W & $15.8^{\mathrm{bc}}$ & 2.9 & 19 & 11.7 & 20.6 \\
PH-LD & $18.0^{\mathrm{c}}$ & 3.4 & 19 & 11.6 & 24.3 \\
\hline
\end{tabular}

* CP: Phosphoric acid, rinse, dry, primer, composite; PA-W: Polyacrylic acid, rinse, wet cotton roll, GIC; PA-D-W: Polyacrylic acid, rinse, dry, wet cotton roll, GIC; PA-LD: Polyacrylic acid, rinse, lightly dry, GIC; PH-W: Phosphoric acid, rinse, wet cotton roll, GIC; PH-D-W: Phosphoric acid, rinse, dry, wet cotton roll, GIC; PH-LD: Phosphoric acid, rinse, lightly dry, GIC.

** SBS with the same superscript letters are not significantly different.

CT group presented bond failures basically in the bracket base-cement interface, while most of the failures with RMGIC were within the cement resulting in some adhesive being left both on the enamel and bracket base.

\section{Discussion}

\section{Comparison Among the PAA Treated Groups}

Moisture contamination resulted in a decrease in SBS of RMGIC, which is in agreement with Rix et al. ${ }^{11}$ and Cheng et al. ${ }^{12}$. Additionally, Cacciafesta et al. ${ }^{13}$ reported higher SBS when enamel was kept moist prior to bonding mechanically retained ceramic brackets with RMGIC. They suggested that moisture contamination appeared to increase the retention of these brackets. Even though the design of the studies was similar, there were some differences that could explain why their conclusions could not be supported by the findings of the present study. While in this experiment human pre-molars were used, Cacciafesta and his coworkers ${ }^{13}$ utilized bovine incisors. They ground the labial surfaces with carbide paper. Therefore, the surface was less irregular, which may have contributed for the higher SBS in their study. Jobalia et al. ${ }^{14}$ reported that moisture was necessary for an ideal performance of Fuji Ortho LC for bonding of orthodontic brackets. However, they used metal brackets and performed a tensile test instead of a shear test.

In this study, two different methods to keep the tooth surface moist were use. The first followed strictly the manufacturer's instructions and consisted in using a moist cotton roll right after rinsing the enamel conditioner. The second method had an additional step that consisted of drying the enamel for $10 \mathrm{~s}$ after rinsing the acid and then applying moisture with a cotton roll. The inclusion of the later group intended to mimic an accidental moisture contamination that is commonly observed in an orthodontic clinic. Although there was no statistically significant difference $(p>0.05)$ between the results of the groups that used these two methods (PA-W, PA-D-W), the first method (PA-W) resulted in lower and more variable SBS values. The variation coefficient of the group that followed the manufacturer's recommendations was $36 \%$, which is considered relatively high. This may be due to the fact that moisture contamination is a difficult variable to control and could become even more unpredictable if tooth surfaces were not dried before the final moisturizing step.

The third group treated with PAA had the enamel slightly dried before bracket placement (PA-LD). It produced SBS values that were higher than both groups bonded with moist enamel (PA-W, and PA-D-W). However, statistically significant difference $(\mathrm{p}<0.05)$ was only from the group that never had the enamel dried after surface conditioning (PAW). Lippitz et al. ${ }^{15}$ reported similar results, suggesting that with a slightly dried surface, RMGIC had SBS equivalent to those observed with CR. However, they used a different storage medium (synthetic saliva), a different RMGIC and metal brackets.

\section{Comparison within the PHA Treated Groups}

Once again, moisture contamination reduced the SBS of RMGIC, but not to a statistically significant level $(p>0.05)$. There were no statistically significant differences $(p>0.05)$ among the three groups with enamel treated with PHA for $15 \mathrm{~s}$. Valente et al. ${ }^{16}$ reported similar results. However, Wiltshire ${ }^{17}$ and Shanilec et al. ${ }^{18}$ reported a significant increase in the retention force of metal brackets bonded with RMGIC after enamel was etched with $35 \%$ PHA for $30 \mathrm{~s}$.

\section{Comparison Between PAA and PHA Groups}

The group treated with PAA that had enamel surface slightly dried presented results that were not statistically different $(p>0.05)$ from any of the groups treated with PHA. The PA-D$W$ group did not have statistically significant different $(p>0.05)$ results from its counterpart treated with PHA.

In addition, the group that followed the manufacturers' instructions (PA-W) had statistically different $(p<0.05)$ results than any of the PHA groups. Overall, there was a tendency to get higher and less variable bond failure loads when PHA was used. As reported earlier, PHA creates 
micropores on the enamel surface that are filled by resin tags increasing the retention of adhesives used in Dentistry. If the resin component of the RMGIC is able to penetrate the porosity created by the acid, mechanical interlock between enamel and the adhesive may happen. Therefore, in addition to the well-reported chemical adhesion of GICs to tooth structure, mechanical adhesion would be obtained resulting in increased SBS. This phenomenon is probably not observed when PAA is applied, because it removes debris and smear layer without creating so many and so deep pores as with PHA.

\section{Comparison of All Experimental Groups to CT}

When RMGIC was used with PHA and the enamel was slightly dried (PH-LD), SBS results were very similar to the CT. The PAA groups bonded with moist enamel (PA-W, and PA-D-W) showed statistically significant lower $(\mathrm{p}<0.05)$ SBS values than CT. Many other studies using not only ceramic, but also metal brackets, have observed similar characteristics ${ }^{19-}$ ${ }^{20}$. In contrast, Cacciafesta et al. ${ }^{13}$ demonstrated that RMGIC had higher SBS than CR when mechanically retained ceramic brackets were used, but not at a statistically significant level. The other experimental groups (PA-LD, PH-W, and PH-DW) presented lower, but not a statistically different $(p>0.05)$ SBS than CP.

\section{Bond Failure Site Comparisons}

An evaluation of the fracture site revealed a statistically significant difference $(\mathrm{p}<0.05)$ among the 7 groups. CT had all the adhesive remaining on the tooth surface on $100 \%$ of the specimens. RMGIC groups with enamel conditioned with PAA showed a tendency to have cohesive failure resulting in most of the cement remaining on the bracket base. About $67 \%$ of the samples in this subgroup had less than $50 \%$ of the adhesive remaining on the enamel. Other $24 \%$ of the specimens had more than $50 \%$ of the adhesive remaining on the teeth, and less than $1 \%$ had all the adhesive remaining either on the tooth or on the bracket base, which is characteristic of an adhesive failure.

RMGIC groups with tooth prepared with PHA presented a performance closer to the one observed with $\mathrm{CR}$, which is in agreement with other studies ${ }^{21}$. This could reinforce the assumption that the percentage of resin material added to the RMGIC could be able to mechanically adhere to tooth structure roughened by the acid conditioning. About $45 \%$ of the failures occurred on the bracket-adhesive interface resulting that all adhesive was left on the enamel.

Other $37 \%$ of the specimens of these three groups had more than $50 \%$ of adhesive remaining on tooth surface, and finally only $18 \%$ of the specimens that used PHA had less than $50 \%$ of the adhesive remaining on the enamel. These results were similar to those reported by Jou et al.22, Haydar et al. ${ }^{23}$ and Summers et al. ${ }^{19}$ who showed ARI (adhesive remnant index) scores similar to those in the present study for ceramic brackets bonded with RMGIC and completely different results for $\mathrm{CR}$ failure. While the present study found that $100 \%$ of the specimens had all the adhesive remaining on the enamel, they found that $80 \%$ of the specimens had the entire adhesive attached to the enamel and the other $20 \%$ had less than $50 \%$ of the adhesive on the tooth. The difference may be explained by the fact that the studies used ceramic brackets with a different retention mechanism.

It may be concluded that: enamel conditioning with $35 \%$ PHA resulted in higher and more consistent SBS values than when PAA was used; moisture contamination significantly decreased SBS of RMGIC on enamel conditioned with PAA and all brackets bonded with composite resin presented adhesive failure at the bracket-adhesive interface, while the RMGIC groups showed a more variable pattern with mostly cohesive failures.

\section{References}

1. da Rocha JM, Gravina MA, da Silva Campos MJ, Quintao CC, Elias $\mathrm{CN}$, Vitral RW. Shear bond resistance and enamel surface comparison after the bonding and debonding of ceramic and metallic brackets. Dent Press J Orthod. 2014; 19: 77-85.

2. Costa AR, Vedovello-Filho M, Correr AB, Vedovello SA, Puppin-Rontani $\mathrm{RM}$, Ogliari FA, et al. Bonding orthodontics brackets to enamel using experimental composites with an iodonium salt. Eur J Orthod. 2014; 36 : 297-302.

3. Ryf S, Flury S, Palaniappan S, Lussi A, van Meerbeek B, Zimmerli B. Enamel loss and adhesive remnants following bracket removal and various clean-up procedures in vitro. Eur J Orthod. 2012; 34: 25-32.

4. Pithon MM, Batista VO, D'El Rey NC. Effect of different methods for decontaminating tooth enamel after contact with blood before bonding orthodontic buttons. J Oral Maxillofac Surg. 2012; 70: 2035-40.

5. da Silva Fidalgo TK, Pithon MM, do Santos RL, de Alencar NA, Abrahao AC, Maia LC. Influence of topical fluoride application on mechanical properties of orthodontic bonding materials under $\mathrm{pH}$ cycling. Angle Orthod. 2012; 82: 1071-7.

6. Kocadereli I, Ciger S. Retention of orthodontic bands with three different cements. J Clin Pediatr Dent. 1995; 19: 127-30.

7. Santos RL, Pithon MM, Fernandes AB, Carvalho FG, Cavalcanti AL, Vaitsman DS. Fluoride release/uptake from different orthodontic adhesives: a 30-month longitudinal study. Braz Dent J. 2013; 24: 410-4.

8. Pithon MM, dos Santos RL, de Oliveira MV, Ruellas AC, Romano FL. Metallic brackets bonded with resin-reinforced glass ionomer cements under different enamel conditions. Angle Orthod. 2006; 76: 700-4.

9. Komori A, Ishikawa $\mathrm{H}$. Evaluation of a resin-reinforced glass ionomer cement for use as an orthodontic bonding agent. Angle Orthod. 1997; 67: 189-95.

10. Artun J, Bergland S. Clinical trials with crystal growth conditioning as an alternative to acid-etch enamel pretreatment. Am J Orthod. 1984; 85: 333-40.

11. Rix D, Foley TF, Mamandras A. Comparison of bond strength of three adhesives: composite resin, hybrid GIC, and glass-filled GIC. Am J Orthod Dentofacial Orthop. 2001; 119: 36-42.

12. Cheng HY, Chen $\mathrm{CH}$, Li CL, Tsai HH, Chou TH, Wang WN. Bond strength of orthodontic light-cured resin-modified glass ionomer cement. Eur J Orthod. 2011; 33: 180-4.

13. Cacciafesta V, Jost-Brinkmann PG, Sussenberger U, Miethke RR. Effects of saliva and water contamination on the enamel shear bond strength of a light-cured glass ionomer cement. Am J Orthod Dentofacial Orthop. 1998; 113: 402-7.

14. Jobalia SB, Valente RM, de Rijk WG, BeGole EA, Evans CA. Bond strength of visible light-cured glass ionomer orthodontic cement. Am J Orthod Dentofacial Orthop. 1997; 112: 205-8.

15. Lippitz SJ, Staley RN, Jakobsen JR. In vitro study of 24-hour and 30-day shear bond strengths of three resin-glass ionomer cements used to bond orthodontic brackets. Am J Orthod Dentofacial Orthop. 1998; 113: 620-4. 
16. Valente RM, De Rijk WG, Drummond JL, Evans CA. Etching conditions for resin-modified glass ionomer cement for orthodontic brackets. Am J Orthod Dentofacial Orthop. 2002; 121: 516-20.

17. Wiltshire WA. Shear bond strengths of a glass ionomer for direct bonding in orthodontics. Am J Orthod Dentofacial Orthop. 1994; 106: 127-30.

18. Shanilec CF, Staley RN, Jakobsen JR. Orthodontic bracket bond strengths with hybrid-glass ionomer cement. J Dent Res. 1998; 77: 298.

19. Summers A, Kao E, Gilmore J, Gunel E, Ngan P. Comparison of bond strength between a conventional resin adhesive and a resin-modified glass ionomer adhesive: an in vitro and in vivo study. Am J Orthod Dentofacial Orthop. 2004; 126: 200-6; quiz 254-5.

20. Godoy-Bezerra J, Vieira S, Oliveira JH, Lara F. Shear bond strength of resin-modified glass ionomer cement with saliva present and different enamel pretreatments. Angle Orthod. 2006; 76: 470-4.

21. Baysal A, Uysal T. Resin-modified glass ionomer cements for bonding orthodontic retainers. Eur J Orthod. 2010; 32: 254-8.

22. Jou GL, Leung RL, White SN, Zernik JH. Bonding ceramic brackets with light-cured glass ionomer cements. J Clin Orthod. 1995; 29: 184-7.

23. Haydar B, Sarikaya S, Cehreli ZC. Comparison of shear bond strength of three bonding agents with metal and ceramic brackets. Angle Orthod. 1999; 69: 457-62. 